\title{
Soin et/ou éducation : au plus près, au plus loin
}

\section{Gérard Fath}

\section{OpenEdition \\ Journals}

Édition électronique

URL : https://journals.openedition.org/leportique/871

DOI : 10.4000/leportique.871

ISSN : $1777-5280$

\section{Éditeur}

Association "Les Amis du Portique"

\section{Référence électronique}

Gérard Fath, "Soin et/ou éducation : au plus près, au plus loin », Le Portique [En ligne], e-Portique, mis en ligne le 08 janvier 2007, consulté le 10 septembre 2022. URL : http://journals.openedition.org/ leportique/871 ; DOI : https://doi.org/10.4000/leportique.871

Ce document a été généré automatiquement le 10 septembre 2022.

Tous droits réservés 


\title{
Soin et/ou éducation : au plus près, au plus loin
}

\author{
Gérard Fath
}

« L'obsession de la démarcation et de la

dichotomie est une des caractéristiques essentielles de l'attitude positiviste » Jacques Bouveresse, Essais IV, Agora, 2004, p.54

1 La complexité de l'éducation suscite fréquemment le recours à la raison graphique (Goody) et typologique et les sciences de l'éducation ne manquent pas de contributions qui distinguent, classent, pour ensuite, réarticuler et décliner des dimensions, dont certaines s'avèrent à l'usage, de véritables items. La rigueur semblerait à ce prix ? Les contributions du dossier préparatoire à ce thème l'auront montré : pour différencier soin et éducation la guerre des paradigmes n'aura pas lieu.

2 Les distinctions typologiques entre les différents schèmes qui composent et traversent le geste éducatif en sa spécificité, n'inclinent pas nécessairement au cloisonnement strict des paradigmes ${ }^{1}$. Une autre voie est possible, que maintes analyses de ce débat illustrent: raisonner en termes de polarités plutôt que de types et de paradigmes, et tracer les lignes de fuite des espaces mentaux ainsi ouverts à l'évolution d'un sujet.

3 Nous suivrons schématiquement cette voie avec l'idée que l'exigence laïque, dont on sait le pouvoir constitutif en France de l'offre éducative, joue à fond de l'ambivalence entre soin et éducation.

4 En effet, du simple fait de l'accueil de tous et chacun, l'école laïque prend soin des singularités, ou du moins, accueille les particularités en les faisant advenir comme singularités à poser dans l'élément de l'universel, à enseigner en un mot. Ce faisant, il est plutôt question d'un champ optimal de médiations pertinentes pour l'évolution d'un sujet que directement d'un sujet apprenant avec qui il faudrait se montrer particulièrement aux petits soins, confusion dont se nourrissent maintes critiques abusives de la pédagogie.

5 En réalité l'effort d'optimisation au quotidien de ce champ de médiations impose aux pédagogues un effort théorique encore méconnu, bien plus vaste et dense que les 
recherches sur la pensée enseignante et le travail enseignant ne le pointent: analyse et composition permanente des médiations selon les multiples dimensions de l'acte en contexte, mais bien plus encore mise en perspective d'horizons et apprentissage de compétences d'investissement sémiotique selon des modes et des registres variés, qu'il importe toujours de garder en visée d'universel.

Cet espace mental qui constitue l'offre éducative, animé par la diversité des rapports au monde mis en œuvre dans l'apprentissage des connaissances, de soi, des autres, se trouve régulé, modalisé au plan axiologique, dans une délicate tension entre le vide et le plein.

7 L'idée d'un vide axiologique sévère, que d'aucuns prescrivent à l'éducation comme gage de neutralité face aux influences, idéologiques et autres, même posée comme condition de l'assomption des Valeurs des Lumières parait ici en échec permanent. L'objectivité des savoirs, la rigueur des argumentations, le courage éclairé des décisions - par-delà l'assaut des opinions et croyances - ne sont-ils pas autant à élaborer, par explicitation et mise à l'épreuve informée des matériaux axiologiques véhiculés, réactivés par les médiations pédagogiques, qu'à stériliser et aseptiser? N’y a-t-il pas là à l'œuvre une authentique négativité philosophique propre à désensabler les positions entre sens et vérité?

8 Art secondaire, dévotion subalterne réputée asservie à l'empirie et à la concrétude approximatives autant qu'intenses, la pédagogie, comme lieu de ce passage exponentiel au plan "méta", ne rejoindrait-elle pas ainsi les préoccupations majeures de la philosophie ? Et si, bien plus, ce passage servait de pierre de touche du soin accordé à l'élan d'êtres singuliers vers l'universel, grâce à la distance récurrente prise à travers l'accueil des phénomènes axiologiques - en somme la course de cet élan - plutôt que du fait de la distance figée, neutraliste, d'un front du refus, d'un glacis inaugural ?

De la typologie des paradigmes aux polarités propres à l'éducation

Simple rappel : le soin spécifique à l'éducation est d'emblée multiparadigmatique. De l'attention vigilante à la prise en charge suivie, de l'intervention clinique de chaque séquence d'enseignement à la veille accompagnant l'observation participante des indices d'appropriation et des effets de transfert escomptés, du souci prégnant pour un tel en particulier à l'attention flottante portant sur des indices d'évolution sur fond d'universel... la liste ouverte est vaste, qui déborde tout tableau cartésien de rangement typologique.

La place nous manque pour examiner comment le soin se module aussi selon les accentuations paradigmatiques internes à la sphère éducation - animer, former, enseigner, instruire.... Bornons-nous-nous ici à quelques traits généraux de ce soin propre à l'éducation.

On peut dire que le soin externe à l'éducation porte davantage sur processus, effets, demande, et, au cœur de l'éducation, plutôt sur les médiations comme telles, les investissements axiologiques et les effets, les compétences acquises sur tout plan et les habitus formés. Alors que le propos du soin en général est la disparition des symptômes, si possible de leurs causes, peut-être l'acquisition de résistances et d'immunité, voire de modes de vie ${ }^{2}$, en éducation, le soin vise l'acquisition d'un potentiel de compétences conférant au sujet une autonomie de développement ouverte au plan culturel en sa généralité et sa complexité, sans inféodation à quelque obédience ou style qui soit, mais pas non plus dans le désert de toute référence, de toute conviction d'ordre existentiel. 
12 Le particulier domine dans le soin en général en tant que mal, souffrance, douleur y sont objectivés comme obstacles à la disposition normale des capacités organiques. Le singulier commande en éducation en tant que, même si l'appropriation y surclasse la simple effectuation normale et particulière, sa dynamique n'est pas centripète, mais heuristique, acheminement vers tout un horizon de mise en jeu de soi, de soi-même comme un autre (Ricœur), et ce dans l'épreuve d'extériorité spécifique de l'école (Marcel Gauchet).

13 Le retour à la norme commande le soin. Non pas certes, au titre d'une conformation étroite - la vie, on le sait, est une sorte de festival, de créativité de normes par ellemême (Canguilhem) - mais au sens où il s'agit de rétablir, par le soin, l'intégrité d'un potentiel organique ou de l'optimiser. L'ouverture heuristique d'horizons de connaissance où la capacité de pensée, de débat argumenté et d'association du sujet se trouve (re)-mise en jeu signe l'éducation.

Soin des médiations, loin des médiations

14 Ce souci des médiations pédagogiques œuvrant à cette ouverture d'horizons ne saurait s'épuiser dans la proximité pédagogique. Il revient en force comme pensée d'un au-delà des médiations qui seraient simplement réglées, domestiquées par quelque institution. Ce mouvement, cet appel d'air depuis l'intérieur le plus confiné de la sphère éducative, se trouve exacerbé par la métaphysique ambiante de la postmodernité et les replis qu'elle opère, de la transcendance sur l'immanence.

15 Les choses de l'éducation et de l'enseignement, en effet, ne seraient guère problématiques s'il suffisait de didactiser convenablement les accès au savoir et ses réélaborations. Nous savons faire, du moins certains le disent. Mais ni épistémologie, ni didactique n'y suffisent. En permanence une nécessité de figuration, d'esthétisation, d'imaginaire autant que d'idéologie traverse et bouscule les progressions dont l'épure, soupire-t-on, rassurait par son efficace, tantôt. On peut gloser indéfiniment sur la sanctuarisation de l'école, nécessaire et/ou impossible, le fait est que les processus d'apprentissage ne se déroulent pas dans une ambiance cognitive aseptisée. L'étiquetage psycho-socio-juridique des perturbations et dysfonctionnements occulte trop souvent le drame de pensée qui se joue là. Que les jeunes êtres en devenir concernés n'aient pas même encore une notion claire de ce que philosopher voudrait dire n'y change rien.

16 Si les simples procédures pédagogiques des médiations pertinentes s'ouvrent sur ce drame de pensée, c'est au sens où l'autonomie de celle-ci s'assure mal quand elle n'est pas assumée à travers ses turbulences et abîmes ${ }^{3}$ auxquels elle se trouve confrontée abîme d'amont en tant que toute pensée s'interroge sur l'énigme de son essor et de sa source, abîme d'aval portant sur l'énigme de ses associations, dérives et variations rebelles à toute enquête définitive, abîme latéral enfin, ouvert par l'alea des reprises pragmatiques de la pensée de chacun par les autres et sa réception de la leur...

17 Si la laïcité est autre chose qu'un souci de neutralité par profil bas des convictions intimes, n'est-elle pas cet espace et cette exigence de soin qui veillerait à l'accueil et à l'élaboration, aussi de l'hétérogène, de l'intempestif, et des lointains de la pensée ?

Le paradoxe relationnel joue ici à fond. On va d'autant mieux au but, supposé et mouvant, que l'on s'éloigne, que l'on intercale, que l'on distancie. Les mouvements pédagogiques l'ont compris et mis en œuvre depuis longtemps, avec leur souci pédagogique d'une diversité et d'une différenciation autant de l'offre des contenus que 
des régimes d'expression sensible, esthétiques, sur fond d'un éventail de régimes de sémiotisation du monde, de soi, de l'autre, et grâce à un travail de modulation des investissements cognitifs selon cette palette d'être au monde.

Il ne suffit pas de concéder cette spécificité du recours aux médiations, dès l'instant qu'il est établi qu'on ne pense jamais sans cheminements et étayages intermédiaires, conscients et/ou inconscients. Il faut de plus prendre la mesure de ce que ce passage méta, du souci de la personne vers le soin apporté au réseau de médiations ne trouve jamais son régime de croisière, ne se laisse caler par aucun expédient technique. Au-delà des aspects et logiques cliniques, le pédagogue réfléchit sur, a le souci de l'éventail des formes et des modes de théorisation même, indéfiniment. Plus encore que continue, l'éducation est récurrente, côté offre comme côté demande.

Y aurait-il ici comme une sorte d'éthique de l'épistémologie dans la recherche des équilibres à ménager entre une démarche mue par une philosophie du concept et une démarche animée par une philosophie du sujet? En effet, faut-il d'emblée se projeter hors immédiateté, pratiquer une pédagogie selon la philosophie de la rupture, du non, et/ou procéder précautionneusement par décrochages successifs, à partir d'un souci foncier d'accueil? De part et d'autre les risques sont légion. Tout pédagogue vit et repense cette tension philosophique plusieurs fois par jour.

21 Entre aménager (des médiations) et ménager (des issues d'altérité, d'universalité, mais aussi des surgissements de subjectivité excédée) ce soin spécifique ne saurait se réduire à quelque routine pédagogique tranquille. Il est le levain dans la pâte de cette routine, dont on sait par ailleurs l'importance, la fonction de cadrage et de trame, aussi bien de repli que d'élan.

Dit autrement, il y a nécessité de prendre soin, en pédagogie, de toute la sphère du symbolico-charnel (Jean-Toussaint Desanti), de toute la contingence anthropologique, non comme de quelque encyclopédie rhapsodique du charnel et de la finitude susceptible de se muer en nouvelle scholastique, comme on le voit, mais en tant qu'elle est source, principe et limite salutaire de toute fonction signifiante, qui s'origine dans ses creux et manques. L'éducation, en tant que pédagogique - plus ou moins, il est vrai, selon ses paradigmes internes - serait donc une sorte de prise en charge soignée de la précarité située en vue d'accès singuliers à l'universel.

Nous empruntons ici la notion de conflit des absolus à Alain Badiou se rapportant à Canguilhem, pour dire qu'il ordonne selon nous, aussi et surtout le champ de travail pédagogique :

(Le sujet vivant est) « impliqué dans les deux termes du conflit. En tant que sujet de la science, il est constituant, par mathématique, expérimentation et technique, de l'universel absolu réel dont tout centre est absent. En tant que sujet vivant, il objecte de cet univers par la singularité mouvante de son milieu propre, centré, normé, signifiant. Dès lors, «sujet » vient nommer en quelque façon, non un des termes de la discordance des absolus, mais plutôt l'énigme de la discordance ellemême. »( Alain Badiou, in Georges Canguilhem, Albin Michel, collectif, pp.297-8, 1993)

Comment la pédagogie ne serait-elle pas contrainte, en ce conflit, par l'exigence et l'obligation de laisser ouvert un milieu d'attente, de remaniement, de veille, de rêverie, de retrait, d'inertie possibles, sorte de chantier de mise au point du rapport au monde selon la pensée réflexive? 
La finitude entre transcendance et immanence

Du coup, soin médical et soin éducatif régissent la finitude en sens opposé: vers la santé comme silence de l'organisme, vers la prise de conscience réfléchie et critique de la relativité (les conditions transcendantales de la connaissance, l'épistémologie en général, mais, surtout, l'exigence laïque de laisser l'espérance en dehors du pré carré de la raison raisonnante et normalisante, ce qui ne veut pas dire la laisser en déshérence, ni surtout refuser de la penser) ${ }^{4}$.

Nous le disions plus haut. Cette pratique réputée simple art, si l'on peut dire, se voit en réalité confrontée à une tache permanente d'arbitrage et de régulation des tensions vers l'immanence et vers la transcendance. Certains rejets purs et simples de la religion et de spirituel en général se traduisant par une récupération lénifiante de la transcendance sur l'axe horizontal, comme le théorise fort bien Luc Ferry ${ }^{5}$, notamment, ne dispensent pas d'un tel travail. Même ainsi repliée, la transcendance reste subversive, ne se laisse pas domestiquer.

Le risque d'une sous-élaboration critique du vide ouvert par réduction de la tension entre ces deux pôles et horizons et de son remplissage par foule de croyances frôlant l'idôlatrie et l'instrumentalisation est on ne peut plus actuel... Il est ainsi des critiques caricaturales des faits de croyance qui veillent à mettre les rieurs de leur côté, et s'abstiennent avec grand soin de se soumettre elles-mêmes au débat raisonné, entretenant, au contraire l'interincompréhension réciproque (Dominique Maingueneau) qui les alimente. Le soin éducatif ici, porterait au contraire sur la prise en charge raisonnée, vigilante, éclairée, de la tension savoir, incroyance, foi (Francis Jacques) par le pédagogue et l'éducation en général. Quelles distinctions, quels points de rencontre entre ces sphères? Peut-on les laisser en état d'indifférenciation critique avec de simples bornages rhétoriques et idéologiques? Comment cerner et valoriser la scientificité même si son rapport aux autres sphères, qu'elle est dite rectifier, déjouer, contenir, n'est pas discerné au plan non pas de l'effectuation méthodologique, mais de ce qui serait une sorte d'épistémologie des croyances en général ?

Double décalage

C'est donc d'un double décalage que nous semble procéder la différence entre soin et éducation, sans que ne cessent les interférences entre les deux gestuelles.

Du soin, centré sur le rétablissement de la personne particulière ou mis à l'exécution d'une tâche, l'éducation déplace l'intention vers les médiations comme étayages vers une connaissance éclairée, source d'autonomie. Mais elle n'en est pas pour autant la science des médiations, qu'il suffirait d'appliquer.

Le second décrochage excentre en effet l'attention aux médiations vers l'attention à leurs pouvoirs d'instauration de mondes possibles.

2 Dire qu'en ce sens toute pédagogie est philosophique, c'est dire qu'il lui importe de veiller à la tension évoquée plus haut, selon les médiations choisies, entre exigences du concept et exigences du sujet, maintien de l'entre-deux, souci permanent de circulation entre ces deux extrêmes, faute de quoi la prise en compte de l'autre se ferait à prisme réduit, étriqué.

Dans la problématique des valeurs ainsi réactivée l'exigence de critères, de jugement, de perspectives s'impose.

L'instrumentalisation - nous parlerions volontiers de gadgétisaton - des valeurs, à la fois esthétisante et mercantile, rend plus que jamais nécessaire, non pas quelque 
retour-à nostalgique, philosophiquement incohérent, mais une remise en perspective des valeurs traversées et véhiculées sur le chemin de connaissance, et ce selon l'exigence laïque. Victor Massuh évoque ainsi cet arrière-plan et cette exigence :

«La colonisation esthétisante du monde par l'œuvre combinée de l'art et de la technique ne se contente pas d'affecter le règne des valeurs, car nous sommes confrontés, sous l'influence conjuguée du nihilisme philosophique et du relativisme éthique, à la notion d'acte gratuit. Celle-ci débouche sur la réactivation rituelle et narcissique du fondamentalisme agressif et désespéré auquel nous assistons.

(...)Nous sommes d'ores et déjà sous le règne du fragment: l'expression des différents styles est devenue cacophonique. (...)

La vie des valeurs aujourd'hui ne nécessite pas d'importants moyens, sinon de la réflexion et du silence. Au-delà des séductions de l'esthétisation, nous devons être réceptifs, sans media ni intermédiaire, à la voix de la vérité, à la sérénité d'une conduite juste et hérö̈que, à l'émerveillement de la beauté et à l'attraction du mystère $»^{6}$

On se rengorgera devant des propos aussi naïfs. Ne convient-il pas aujourd'hui d'exhiber chacun sa (post)modernité soit en criant sus aux valeurs, soit en s'ébattant dans leur foisonnement? Veiller à toute capture de cette voix de la vérité ne dispense pas mais exige de différencier sens et vérité pour la rigueur même du jugement critique. Pour nous, la différence entre soin et éducation est ici pointée.

De la citation de Massuh, précisément sur fond de cette aspiration naïve en apparence, nous retenons l'incontournable souci de perspective, de cohérence au plus haut niveau, par-delà les harmonisation rhétoriques et idéologiques. C'est dit avec des mots d'autrefois? L'exigence demeure actuelle: au-delà de tout soin qui rétablisse, $\hat{o}$ combien utilement, l'éducation met en perspective, développe la capacité à identifier, analyser, critiquer les horizons ouverts, mais bien avec le souci d'interroger leur habitabilité, leur accès pour tous et chacun.

Exigence double, en effet. Si précieuse que soit aujourd'hui la voie érotétique creusée par les travaux méticuleux d'un Francis Jacques dégageant la spécificité interrogative des postures de croyance, il revient spécifiquement à l'exigence laïque d'articuler ces modes de questionnements aux mondes possibles afférents, à travers une phénoménologie du séjour pertinente, encore à dégager des errements et des vertiges de l'immanence qui la saisissent. La tâche est rude, mais nécessaire, car, s'il revient à tout sujet d'inscrire ses exigences de vérité, ou l'exigence de ses vérités, dans le réel, il le fera, certes selon sa liberté plénière, mais aussi sur fond de l'éducation qui lui aura permis de se poser face à ces vérités et de vivre selon celles-ci. La spécificité transcendantale des croyances relatives aux fins dernières, qu'il est pertinent de situer dans le questionnement plutôt que dans l'effectuation servile, ne se soutient pas sans la quête laïque d'un monde co-habitable, que l'éducation, tout particulièrement laïque, se doit de nourrir et de préserver de toute capture et clôture.

D'où l'importance, à l'image de Ricœur, de se maintenir entre critique et conviction.

40 conceptions de la philosophie $e^{7}$ évoquées plus haut. Le terrain de cette tension, n'est-ce pas la laïcité, toujours à nouveau à creuser?

41 Pour nous, une laïcité repensée correspond au soin mis à faire vivre cette tension tout en gardant les droits de l'espérance. Nous la concevons en ce sens comme une théorisation faible, opératoire comme telle, de l' Espace Axiologique qu'engendre tout 
geste éducatif d'importance ${ }^{8}$. Autant que le metteur en œuvre s'en avise et veille à réélaborer au mieux cet espace.

Ce souci de maintenir vivante et habitable la tension évoquée se redouble en nécessité de vigilance, de tact, de pondération sans capitulation: éviter que le passage exponentiel au plan méta ne s'emballe au point de susciter vertige et délire chez des êtres fragiles en évolution. Précisément, la laïcité est aussi cette exigence de non exacerbation des visées d'horizons. Qu'elle soit le fait des hymnes inconditionnels au progrès par les Lumières ou des exaltations de visionnaires instrumentalisant l'accès automatique à quelque paradis qui soit, importe peu.

Ne jamais affronter ces extrêmes au cœur de l'éducation expose, faute de connaissances historiques et de débats à propos des utopies, des idéologies de masse, des révolutions de mentalités, tout autant que des religions...) à l'implosion individualiste et consommatrice entreprise par une société de l'image, de la rumeur, du fantasme, organisée en marché de l'égarement ${ }^{9}$.

Qu'est-ce qu'une société qui voudrait tout régler jusqu'à exclure désir, hubris, ruptures de vérités par ses soins? Mais aussi qu'est-ce qu'une société qui prétendrait faire accéder au savoir sans prendre aucun soin de ses appropriations et surdéterminations singulières?

La pédagogie ne doit-elle pas rejeter ce soin-là, qui conditionne - fût-ce au bonheur euphorisant des certitudes closes - mais prendre soin de tout ce qui permet d'accéder à l'universel singulier par-delà l'atomisation particulariste?

L'exigence laïque, travail à la fois compréhensif et critique sur un espace axiologique des modes et contenus d'investissement de sens, nous semble, de ce point de vue, à la fois à retrouver et à repenser... avec soin.

\section{NOTES}

1. Ni même à la distinction de plans "irréductibles avec leurs éléments ", telle que la propose Deleuze in Qu'est-ce que la philosophie, ed.Minuit 1991,p.204. En éducation, concept, sensation, connaissance s'entremêlent et s'étayent constamment, de même qu'affects, percepts, concepts ne s'offrent pas par plans entiers de consistance homogène, mais se dispersent selon la logique de découverte existentielle. Ce qui est à moduler et à élaborer ce n'est pas la consistance de chaque plan (plan d'immanence de la philosophie, plan de composition de l'art, plan de référence ou de coordination de la science, ib.), mais les investissements et instaurations axiologiques de ces plans à travers les tâtonnements d'un devenir.

2. Cette tendance à glisser de la thérapie à l'option existentielle, voire à une vision du monde globale, s'observe de plus en plus, notamment à connotation écologiste. Abondamment discouru, pourvu de sa rhétorique, ce glissement est-il toujours analysé dans ses implications tout autres que médicales?

3. BELLET, Maurice in Critique de la raison sourde, pp.32 sq., Desclée de Brouwer, 1992 
4. Reclus dans sa chambre, le poête Jöe Bousquet n'était pas en bonne santé. Au plan métaphysique, accessible par la parole poétique, il y avait là plus que de la santé. D'autres exemples viennent à l'esprit, dont la pédagogie peut avoir l'intelligence et l'usage.

5. L'homme-dieu ou le Sens de la vie, Grasset, 1996.

6. MASSUH, Victor, L'âme des valeurs, in Où vont les valeurs ?, coll., s/dir. Jérôme Bindé, Ed. de l'Unesco, Albin Michel, 2004, pp.129-129

7. telles que les commente BOUVERESSE, Jacques, in Essais IV, Pourquoi pas des philosophes, , pp.196, sq. Agone, 2004.

8. Fath, Gérard in Ecole et valeurs : la table brisée ?, Lharmattan, 2006.

9. Il n'est pas sûr qu'une pédagogie du fait religieux, conçu comme quasi discipline réponde à cet enjeu.

\section{RÉSUMÉS}

Pratique réputée simple art, au mieux, la pédagogie se voit en réalité confrontée à une tache permanente d'arbitrage et de régulation des tensions vers l'immanence et vers la transcendance. Elle génère nécessairement la pensée d'un au-delà de toutes médiations en tant qu'elles seraient simplement réglées, domestiquées par les attentes et les normes qui cristallisent autour de quelque institution. Cet appel d'air depuis l'intérieur le plus confiné de la sphère éducative, se trouve exacerbé par la métaphysique ambiante de la postmodernité et les replis, qu'elle opère, de la transcendance sur l'immanence. La problématique laïque s'en trouve relancée.

Si la laïcité est autre chose qu'un souci de neutralité par profil bas des convictions intimes, n'estelle pas cet espace et cette exigence de soin qui veillerait à l'accueil et à l'élaboration critique, aussi de l'hétérogène, de l'intempestif, et des lointains de la pensée?

Il reviendrait alors aux pédagogues, par-delà toute technicité, de s'équiper pour élaborer avec soin une sorte d'éthique de l'épistémologie dans la recherche des équilibres à ménager entre une démarche mue par une philosophie du concept, qui interpelle les évidences natives et une démarche animée par une philosophie du sujet, qui suscite une herméneutique de l'élan singulier vers la vérité.

Pedagogy is not merely an art of teaching. It has to arbitrate and to regulate constantly between immanence and transcendance. So its purpose is to think beyond all mediations which are controlled by expectations and standards crystallized by institutions. Postmodernity exacerbates this orientation by reducing somehow transcendance to immanence. In this way the question of laicity becomes afresh problematic.

If laicity is not simply neutral out of indifference to personnal convictions, it must be a frame in which thinking has to be welcome and worked out in a critical way through all its various aspects and distant views.

Beyond all their technical skill, teachers, have, therefore, to prepare themselves in order to build a kind of ethical epistemology able to balance philosophy of concept and philosophy of subject. 


\section{AUTEUR}

\section{GÉRARD FATH}

Agrégé de Philosophie, ancien Elève de l'Ecole Normale Supérieure de Saint-Cloud, Docteur d'Etat, Gérard FATH est actuellement Professeur émérite de l'Université Nancy 2. Successivement enseignant de philosophie, formateur d'enseignants spécialisés, enseignant-chercheur en Sciences de l'Education, il explicite ici les deux thèses qui constituèrent la trame d'interventions diverses auprès de publics très variés. Le chantier ouvert mène, plus loin, vers le statut de la croyance en général dans l'acte éducatif, avec ses impacts sur les philosophies du sujet, des valeurs, du langage, notamment. 